\title{
An Accelerated 3D-Magnetostatic Field Computation Method With Tetrahedral Surface Integrals
}

\author{
Wenchun Zhao ${ }^{1}$, Wubing $\mathrm{Zhu}^{1^{*}}$, Jinwu Zhuang ${ }^{1}$ \\ 1.Electrical Engineering College, Naval University of Engineering, Wuhan 430033, Chin) \\ * Corresponding author: Wubing Zhu, Email: zhuwubing2014@hotmail.com
}

Keywords: integral equation method; tetrahedral surface; shared and non-shared face; accelerated method

\begin{abstract}
An innovative accelerated integral equation method is proposed based on a fast search algorithm for both shared and non-shared faces among mesh elements. This method successfully accelerates the computational process of solving for the tetrahedral coupling coefficients. By using the proposed accelerated integral equation method, the efficiency of constructing the coupling matrix can be improved by more than $40 \%$ without decreasing computational accuracy, as demonstrated by analytical solutions, simulations and experimental examples.
\end{abstract}

\section{Introduction}

Induced magnetism, caused by the movement of ships, planes, and other metallic vehicles within a magnetic field, is one of the main threats to the security of naval vessels $[1,2]$. It is also a primary factor hindering the development of highprecision magnetic measurement technologies using sensors aboard these vehicles [3]. With regard to ship degaussing [4, 5], mastering the characteristics of a vessel's induced magnetic field is vital to realizing effective compensation methods. Marine and aerial magnetic surveys also suffer from induced magnetic fields as one of the main sources of interference [6, 7]. Due to the complex geometric features of vehicles, numerical methods, such as the finite element method (FEM) [8], the boundary element method (BEM) [9] and the integral equation method (IEM) $[10,11]$ have been effective tools for calculating the induced magnetism accurately. IEM may be more suitable for open-boundary magnetostatic field calculations that avoid meshing the air region (or the equivalent) [10-13].

The numerical modeling of the induced magnetism caused by vehicles has resulted in a series of developments using IEM. In Ref. [14], by utilizing the surface integral calculation method and comparing the results with the volume integral method, the computation of the coupling coefficient matrix is simpler. In addition, the singularity in the integral is eliminated, while in the IEM models, the non-symmetric and dense coefficient matrix results in low computation efficiency.
To overcome the disadvantages described above, accelerated approaches, such as ACA [15], FMM [11, 16] and the parallel computing method [17], have been applied to successfully realize large-scale magnetism computations. The ACA, FMM, and the parallel computing technique for IEM method are three independent methods (the parallel computing method can also be intergrated with the ACA or the FMM) for accelerating the magnetostatic field computation, and the memory cost can also be reduced by the three methods simultaneously. In the three fast computational methods above, considerable amounts of surface integrals are needed to complete the computational process. However, the repeated calculations of the surface integral of shared faces among adjacent elements has so far been ignored, an innovative accelerated method based on a fast search algorithm [18] for shared and non-shared faces is proposed here, and the proposed accelerated would be possible used to accelerate ACA, FMM and parallel computing technique for IEM furtherly in future work.

In Section 2, the vector integral equation method based on a tetrahedral mesh is introduced. In Section 3, the theory behind the reduction of surface integral calculations will be demonstrated, and an approach for accelerated IEM based on a fast algorithm [18] for searching shared and non-shared faces will be proposed. Then, in Section 4, the analytical calculation of a sphere magnetized by a uniform applied magnetic field will be provided, along with a model for representing the magnetostatic fields of an irregular ferromagnetic object and a thin steel plate placed in various magnetic environments. These examples will help demonstrate the advantages and accuracy of the proposed accelerated IEM models.

\section{Vector integral equation method}

As shown in Figure 1, according to the IEM, when a ferromagnetic object is placed in a geomagnetic field, the induced magnetic flux density $\boldsymbol{B}$ at an arbitrary point $P$ outside the object can be expressed as [19]

$$
\boldsymbol{B}\left(\boldsymbol{r}_{\boldsymbol{P}}\right)=-\frac{\mu_{0}}{4 \pi} \nabla_{\boldsymbol{P}} \int_{V} \boldsymbol{M}\left(\boldsymbol{r}_{Q}\right) \cdot \nabla_{\boldsymbol{Q}}\left(\frac{1}{\left|\boldsymbol{r}_{\boldsymbol{P} Q}\right|}\right) \boldsymbol{d} \boldsymbol{v}_{\boldsymbol{Q}}
$$


where $\boldsymbol{M}$ denotes the magnetization of the ferromagnetic object, $\boldsymbol{r}_{P Q}$ represents the vector from the source point $Q$ to the field point $P, \mu_{0}$ represents the permeability of free space, $\nabla_{p}$ and $\nabla_{Q}$ represent the gradient operator to the field coordinate and the source coordinate, respectively, and $V$ represents the volume of the object.

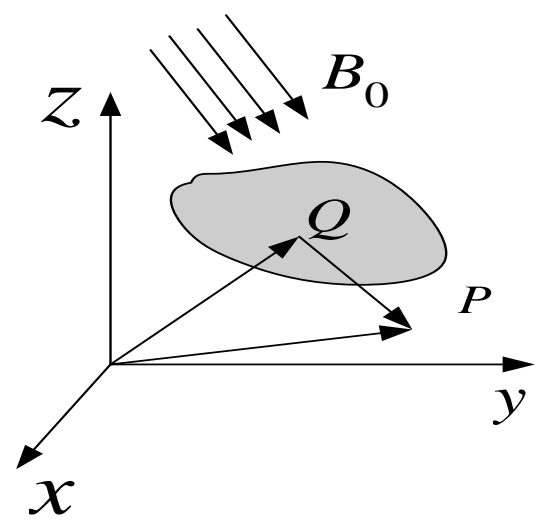

Figure 1 Sketch of a magnetostatic computation

To calculate the induced magnetic flux density $\boldsymbol{B}$ created by the ferromagnetic object, the object should be discretized into $N$ elements that may be tetrahedral in shape. Eq. (1) becomes,

$$
\boldsymbol{B}\left(\boldsymbol{r}_{\boldsymbol{P}}\right)=-\frac{\mu_{0}}{4 \pi} \sum_{i=1}^{N} \nabla_{P} \int_{V_{i}} \boldsymbol{M}\left(\boldsymbol{r}_{Q}\right) \cdot \nabla_{Q}\left(\frac{1}{\left|\boldsymbol{r}_{P Q}\right|}\right) \boldsymbol{d} \boldsymbol{v}_{Q}
$$

As far as the magnetization of each element is concerned, the constant, linear, and higher order shape functions can be used. For simplicity, the constant shape function is used in this computation, Then the field created by each volume element can be calculated using the equivalent surface distribution method [13],

$$
\boldsymbol{B}\left(\boldsymbol{r}_{\boldsymbol{P}}\right)=-\frac{\mu_{0}}{4 \pi} \sum_{i=1}^{N}\left(\nabla_{\boldsymbol{P}} \int_{S_{i}} \frac{\boldsymbol{M}_{i}\left(\boldsymbol{r}_{Q}\right) \bullet \boldsymbol{n}_{i}}{\left|\boldsymbol{r}_{P Q}\right|} d \boldsymbol{S}_{Q}\right)
$$

where $S_{i}$ represents the surface area of the volume element and $\boldsymbol{n}_{\boldsymbol{i}}$ represents the direction normal to the surface. When computing the induced field created by an external magnetic field, the object is usually divided into a sufficient number of uniform elements. Considering the relationship $\boldsymbol{M}=\boldsymbol{B}\left(\mu_{r}-1\right) / \mu_{r}$ and each of the $N$ field points that are placed at the barycenter of the corresponding tetrahedral element, the following system can be obtained,

$$
\begin{aligned}
& \frac{\mu_{r j}}{4 \pi} \sum_{i=1}^{N} \frac{\mu_{r i}-1}{\mu_{r i}} \oint_{s_{i}}\left(\boldsymbol{B}\left(\boldsymbol{r}_{Q_{i}}\right) \cdot \boldsymbol{n}_{i}\right) \frac{\boldsymbol{r}_{P Q_{i}}}{\left|\boldsymbol{r}_{P Q_{i}}\right|^{3}} d s_{Q}-\boldsymbol{B}\left(\boldsymbol{r}_{P_{j}}\right) \\
& =-\mu_{r j} \boldsymbol{B}_{0}\left(\boldsymbol{r}_{P_{j}}\right)
\end{aligned}
$$

where $i$ and $j$ represent the element numbers while $\mu_{r i}$ and $\mu_{r j}$ denote the relative permeability of respective tetrahedral elements. Compared with other strong magnetic fields, the geomagnetic field is so weak that the relative permeability $\mu_{r}$ can be treated as a constant. Eq. (4) can then be transformed into a matrix form as follows

$$
\begin{aligned}
& \frac{\mu_{r j}}{4 \pi} \sum_{i=1}^{N} \frac{\mu_{r i}-1}{\mu_{r i}} \boldsymbol{S}_{P_{j} Q_{i}} \boldsymbol{B}\left(\boldsymbol{r}_{Q_{i}}\right)-\boldsymbol{B}\left(\boldsymbol{r}_{P_{j}}\right) \\
& =-\mu_{r j} \boldsymbol{B}_{0}\left(\boldsymbol{r}_{P_{j}}\right)
\end{aligned}
$$

After solving the matrix system above, the magnetic flux density of each tetrahedral element can be obtained. The induced magnetic flux density $\boldsymbol{B}$ at an arbitrary point $P$ around the ferromagnetic object can be calculated based on Eq. (3).

\section{Accelerated 3D-Magnetostatic Field Computation Method}

According to Eq.(5), Solving the coefficient matrix $\boldsymbol{S}_{P_{j} Q_{i}}$ associated with each tetrahedral element is central to the problem of obtaining the internal magnetic induction $B_{i}$ of each mesh element. To obtain the coefficient matrix, the integrated contributions to the match point (barycenter of each volume element is selected as the match point) of the four faces of each element must be calculated. Here, the ferromagnetic object is divided into tetrahedrons, and two ordinary elements, element $\boldsymbol{A B C D}$ and element $\boldsymbol{A C D E}$, are demonstrated in Figure 2. An example of $\boldsymbol{S}_{P_{j} Q_{i}}$ are calculated with the two elements above,

$$
\boldsymbol{S}_{P_{j} Q_{i}}=\oint_{S_{i}} \frac{\boldsymbol{r}_{P Q_{i}}}{\left|\boldsymbol{r}_{P Q_{i}}\right|^{3}} \cdot \boldsymbol{n}_{i} d s_{Q}=\sum_{l=1}^{4} \oint_{s_{l}} \frac{\boldsymbol{r}_{P Q_{i}}}{\left|\boldsymbol{r}_{P Q_{i}}\right|^{3}} \cdot \boldsymbol{n}_{i} d s_{Q}
$$

where $S_{i}$ represents the surface area of the volume element $\boldsymbol{A C D E}, \boldsymbol{n}_{\boldsymbol{i}}$ represents the direction normal to the surface, $S_{l}(l=1,2,3,4)$ represent the four triangle surfaces of the volume element $\boldsymbol{A C D E}, \boldsymbol{r}_{P Q_{i}}=\left(x_{P}-x_{Q_{l}}\right) \boldsymbol{i}+\left(y_{P}-y_{Q_{1}}\right) \boldsymbol{j}+\left(z_{P}-z_{Q_{l}}\right) \boldsymbol{k}$, $\left(x_{P}, y_{P}, z_{P}\right)$ is the barycentric coordinate of the volume element $\boldsymbol{A B C D}$. Thus,

$$
\begin{aligned}
& s_{l x}=\oint_{s_{l}} \frac{x_{P}-x_{Q_{l}}}{\left|\boldsymbol{r}_{P Q_{l}}\right|^{3}} d s_{Q} \\
& s_{l y}=\oint_{s_{l}} \frac{y_{P}-y_{Q_{l}}}{\left|\boldsymbol{r}_{P Q_{l}}\right|^{3}} d s_{Q} \\
& s_{l z}=\oint_{s_{l}} \frac{z_{P}-z_{Q_{l}}}{\left|\boldsymbol{r}_{P Q_{l}}\right|^{3}} d s_{Q}
\end{aligned}
$$


Surface integrals $s_{l x}, s_{l y}$ and $s_{l z}$ can be computed analytically or by using numerical Gauss technique to obtain $S_{P_{j} Q_{i}}$. The computation of the surface integral over $\boldsymbol{A C D}$ is required for calculating the coefficient matrices for both elements $\boldsymbol{A B C D}$ and $\boldsymbol{A C D E}$, and case is the same when the coupling matrice between one of the two elements above and any one of other elements are calculated. Because the normal vectors for the two volume elements point in the opposite direction, the contribution from face $\boldsymbol{A C D}$ on the given point will be opposite. By calculating and saving the surface integrals of all the shared faces (number of surface integrals of all the shared faces can be reduced by 50\%) and nonshared faces firstly, efficiency of the coefficient matrices calculation can be improved.

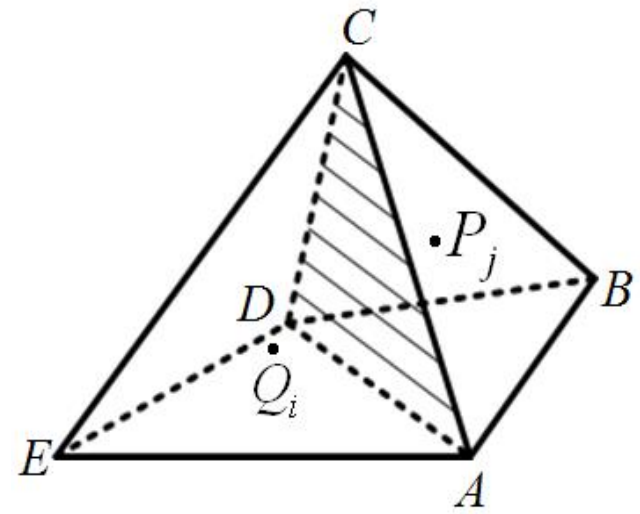

Figure 2 Neighboring tetrahedral elements of a ferromagnetic object

\subsection{Fast search algorithm for shared and non-shared faces}

Ferromagnetic objects can be meshed with Gmesh, Hypermesh or other mesh tools from commercial FEM software. Here, the mesh tool of the Flux3D program is used to mesh ferromagnetic objects. Two types of mesh data can be generated, either (1) the number and coordinates of nodes or (2) the number of mesh elements and associated nodes. A list of shared and non-shared faces can be acquired from these data. In fact, all the information needed regarding shared and non-shared faces can be found by pairwise comparison. However, because pairwise comparison is not efficient, a fast search algorithm [18] based on the concepts and principles of adjacency and incidence matrices for graph can be applied to identify the two types of faces. Because of the search process implemented with the transpose and multiplication for sparce matrice, the computational complexities and storage complexities of the search algorithm is $\boldsymbol{O}(\boldsymbol{N})$. At the same time, the shared and non-shared faces are numbered for the sake of using them to accelerate the conventional IEM[13, 20].

\subsection{Accelerated method for IEM}

Based on the information of shared and non-shared faces, an accelerated method for IEM is proposed. In fact, the computational efficiency of the accelerated IEM is reflected by the calculation of coefficient matrices. Here, the computational procedures of the accelerated IEM are demonstrated in Figure 3. This accelerated IEM program uses MATLAB 2014 and was tested on a PC with an Intel Core (TM) i7-3770 CPU at $3.40 \mathrm{GHz}$ and $16 \mathrm{~GB}$ RAM.

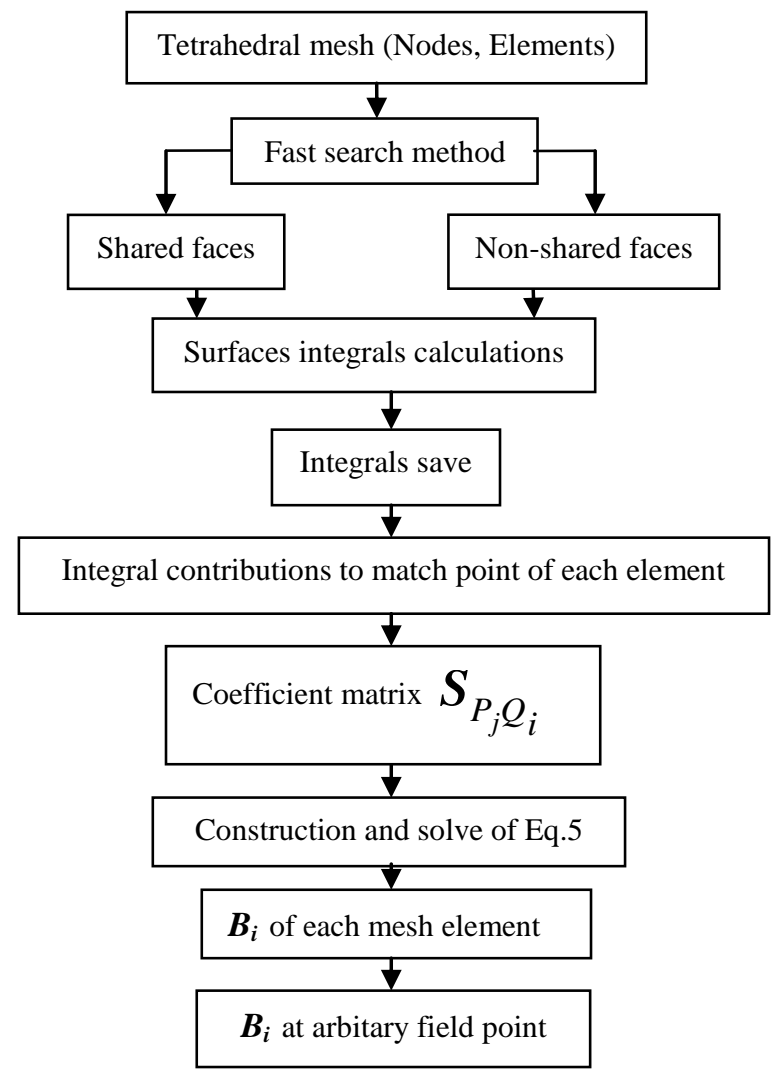

Figure 3 Flowchart of the proposed accelerated IEM

\section{Calculations and verifications}

Three examples are provided here to demonstrate the proposed accelerated IEM algorithm. The first example is an analytical example where the induced field of a sphere is placed in a uniform external magnetic field. The second example is the magnetostatic modeling of an irregular object magnetized by an applied uniform external field. Finally, a simple experiment is executed with a steel plate to verify the numerical results of the magnetostatic field calculations using IEM acceleration.

\subsection{Analytical example}

An iron sphere with a radius of $50 \mathrm{~mm}$ and a relative permeability of 150 is placed into an external uniform field with $B_{0}=34500 e_{z} n T$. In computing the induced field caused by the ferromagnetic sphere, 101 spots are arranged along line 1 (as shown in Figure 4, from point P1 to point P2) equally. The analytical solution can be obtained following Ref. [20], which will be compared with the calculated results of the proposed method. The iron sphere is discretized with either 257, 499, 945, or 1250 nodes in four separate calculations. In each mesh condition, the induced field at all 
spots is calculated by both conventional IEM and the proposed accelerated IEM. As shown in Table. 1, the numbers of mesh nodes $(N 1)$, elements (N2), public faces (N3), nonpublic faces (N4), total faces (N5), computational time $t 1$ (conventional IEM), and computation time $t 2$ (accelerated IEM) of the four mesh cases are displayed. For accelerated IEM, the reduction of the number of surface calculation required can greatly speed up matrix construction. In the four mesh conditions, the ratios of surface integrals needed for accelerated IEM and conventional IEM are 1533/2588, $4370 / 8176,8819 / 16724$ and 11904/23040. The ratios of computation time for matrix construction between accelerated IEM and conventional IEM are 358.3/579.4, 3218.2/5715.1, 12848.1/26394.9 and 22995.35/54420.7, which demonstrates the efficiency of our proposed accelerated IEM technique.

The graphs for the induced magnetic field strength using the analytical solution (labeled Analytical×), conventional IEM (labeled NORMALX), and the proposed algorithm (labeled FAST $\times$ ) with $257,499,945$, or 1250 nodes are shown in Figures. 5-7 and Figure13. As the number of mesh nodes increases, the magnetic flux, $B_{z}$, calculated using either accelerated or conventional IEM approaches the analytical solutions. In Figures 8, 10 and 12, the X-component, Ycomponent, and Z-component of the induced magnetic flux intensity $\left(B_{x}, B_{y}, B_{z}\right)$ are displayed, respectively, and the errors of the three components at each calculated point are shown in Figures 9, 11 and 13, respectively. The errors in $B_{x}$ and $B_{z}$ are below $1.5 \%$, and the errors of $\left|B_{y}\right|$ are less than $2 n T$ (analytically, $B_{y}$ is zero at any point in Line 1 ).

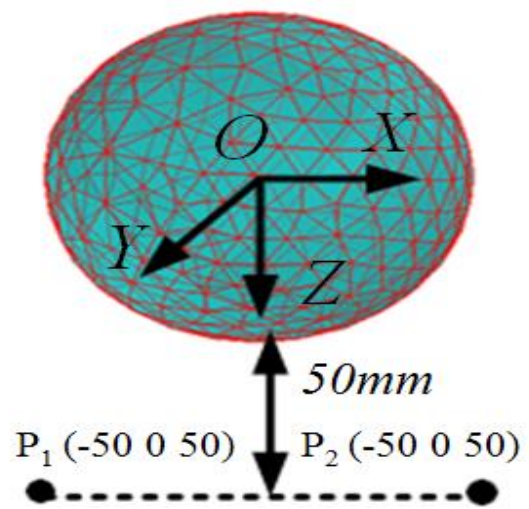

Figure 4 Map of the ferromagnetic solid sphere

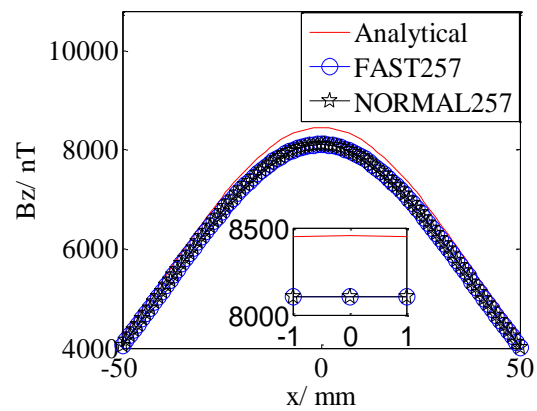

Figure 5 Magnetic flux density Bz with 257 nodes

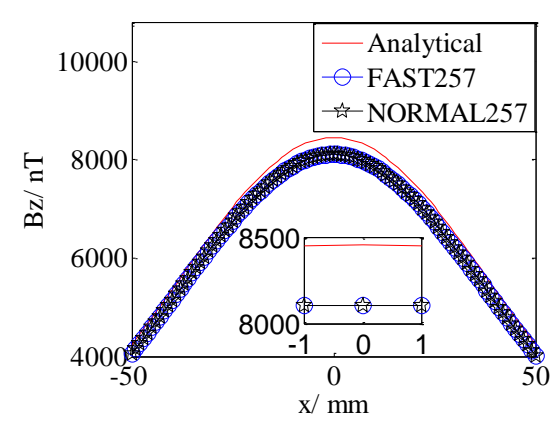

Figure 6 Magnetic flux density Bz with 499 nodes

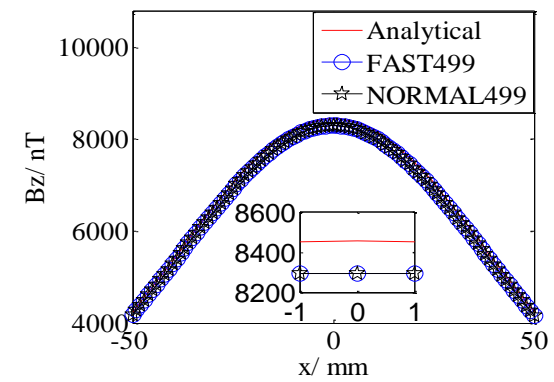

Figure 7 Magnetic flux density Bz with 945 nodes

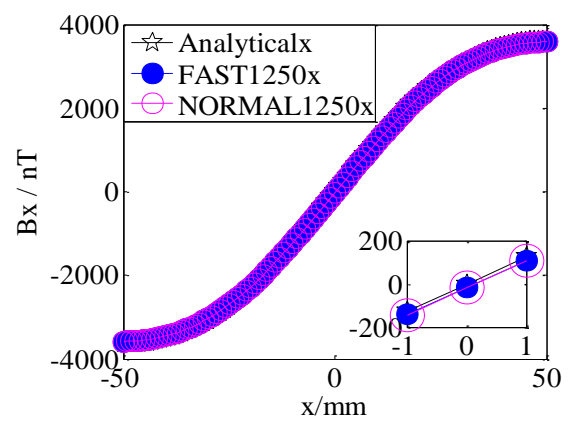

Figure 8 Magnetic flux density Bx with 1,250 nodes

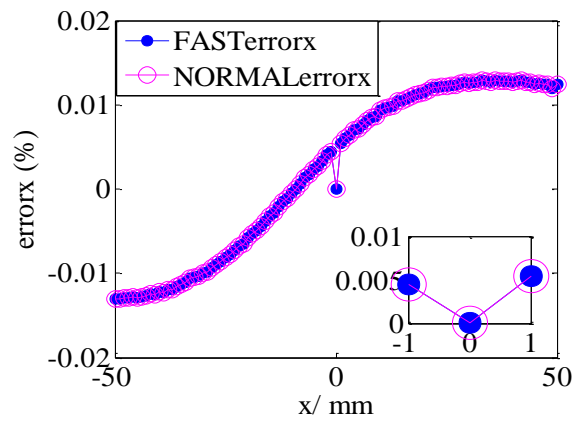

Fig. 9 Error in Bx with 1,250 nodes

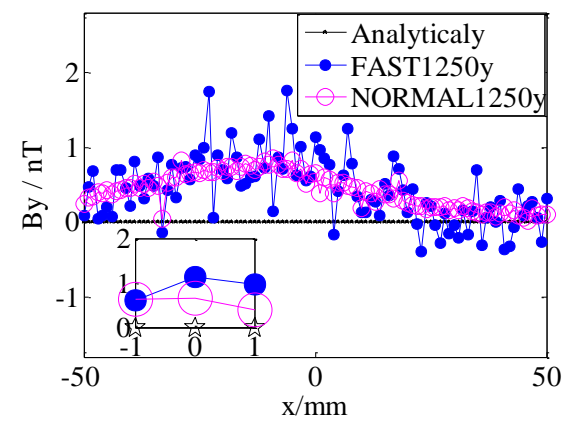

Figure 10 Magnetic flux density By with 1,250 nodes 


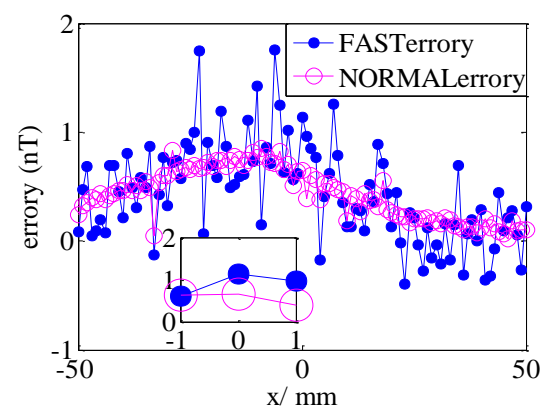

Figure 11 errors of By with 1,250 nodes

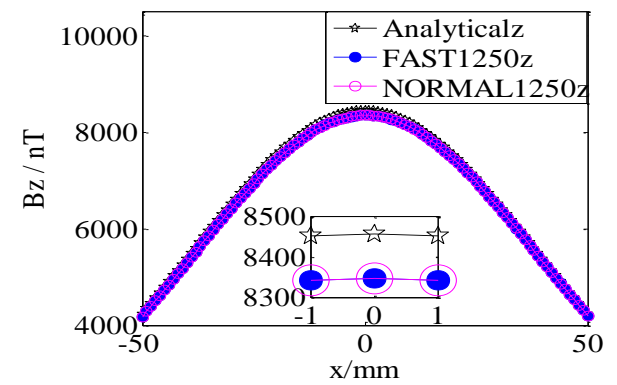

Figure 12 Magnetic flux density Bz with 1,250 nodes

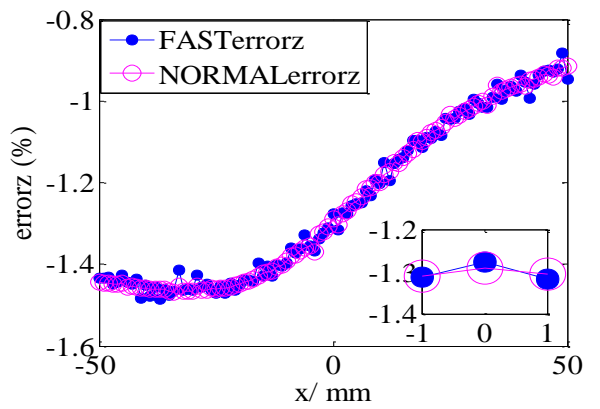

Figure 13 errors of $\mathrm{Bz}$ with1,250 nodes

Table. 1 parameters of the conventional IEM and the

\begin{tabular}{|l|l|l|l|l|l|l|}
\hline$N 1$ & $N 2$ & $N 3$ & $N 4$ & $N 5$ & $t 1 / s$ & $t 2 / s$ \\
\hline 257 & 647 & 1055 & 478 & 1533 & 579.4 & 358.3 \\
\hline 499 & 2044 & 3806 & 564 & 4370 & 5715.1 & 3218.1 \\
\hline 945 & 4181 & 7905 & 914 & 8819 & 26394.9 & 12848.1 \\
\hline 1250 & 5670 & 10776 & 1128 & 11904 & 54420.7 & 22995.4 \\
\hline
\end{tabular}

\subsection{Simulation example}

As shown in Figure 4, a solid, irregularly shaped iron object with a relative permeability of 150 is placed into an external uniform field $B_{0}=34500 e_{z} n T$. The coordinates of the points in Fig. 14 are $\boldsymbol{A}(-10,-50,-10), \boldsymbol{B}(-10,-50,10)$, $\boldsymbol{C}(10,-50,-10), \boldsymbol{D}(10,-50,10), \boldsymbol{E}(10,20,10)$, $\boldsymbol{F}(0,80,0), \boldsymbol{P}_{1}(0,-100,20), \boldsymbol{P}_{2}(0,100,20)$. The 201 field points are arranged equally along Line 2 (as shown in Figure 14, extending from P1 to P2). Using the mesh tool of Flux3D with 1061 nodes and 3739 elements, the three components of the magnetic flux of the irregular object are calculated by the conventional method and the proposed accelerated method. For conventional IEM, the number of surface integrals is 14596 , and it takes 20296.7 s to construct the system matrix. On the other hand, the accelerated IEM uses 8262 surface integrals and can construct the system matrices utilizing 6694 shared faces and 1568 non-shared faces in $12163.1 \mathrm{~s}$, which is approximately $60 \%$ of the time required for the conventional method.

In order to verify the accuracy of the accelerated IEM algorithm, each point was also calculated using Flux3D, and the results were compared with those from conventional IEM and the proposed accelerated IEM. The curves, the flux (labeled Flux $x$ ), the conventional IEM (labeled NORMALx) and the accelerated IEM (labeled FAST $\times$ ) are provided in Figures 15-17. Here, the values of the Flux3D simulations are taken as the baseline. The maximal errors of $B_{y}, B_{z}$ with the conventional IEM and the accelerating IEM are $2.31 \%$, and the errors of $\left|B_{y}\right|$ are less than $15 n T$ (the analytical solutions $B_{y}$ of each calculating field point is zero), which demonstrates the accuracy of the proposed accelerated method.

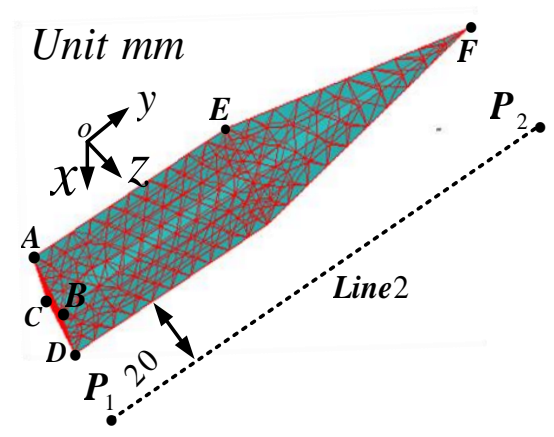

Figure 14 Map of the solid irregular object

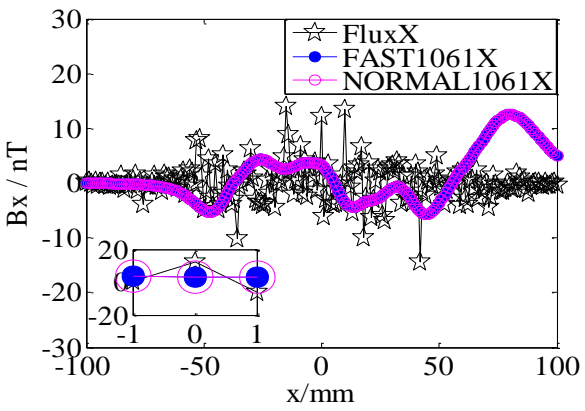

Figure $15 \mathrm{Bx}$ of the solid irregular object

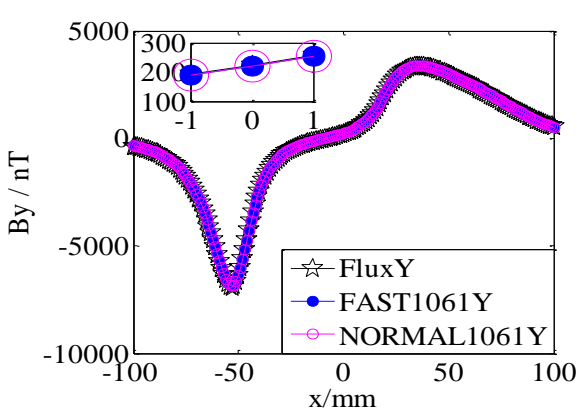

Figure 16 By of the solid irregular object 


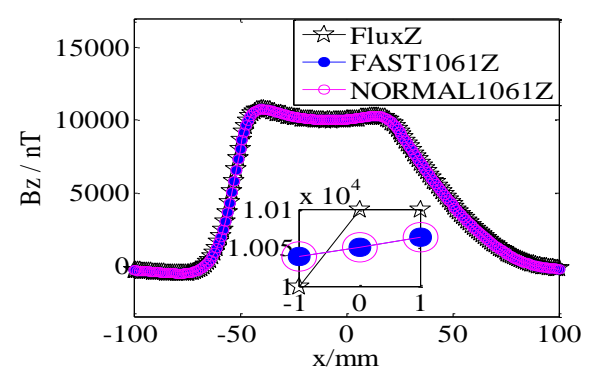

Figure $17 \mathrm{Bz}$ of the solid irregular object

\subsection{Experimental example}

As shown in Figure 19, a steel plate with a relative permeability of 100, a length of $1000 \mathrm{~mm}$, a width of $200 \mathrm{~mm}$, and a thickness of $27 \mathrm{~mm}$ is placed in a geomagnetic field with a horizontal component of $34484.6 \mathrm{nT}$ and a vertical component of $34800 \mathrm{nT}$. The 31 measured points are located uniformly along Line 3 (from $\mathrm{P}_{1}$ to $\mathrm{P}_{2}$ ). In the laboratory (Figure 18), the steel plate is placed on a nonmagnetic carrier on a track. The axes of the triaxial magnetic sensors are aligned along the same as the steel plate coordinates system shown in Figure 19, and the sensor is located on Line 3 to provide reference values. When the nonmagnetic carrier moves over the magnetic sensors, the magnetic anomaly of the steel plate can be recorded. To eliminate the influence of the permanent magnetization, the steel plate is placed in four different orientations. Based on the measured field in the four orientations, the longitudinal and vertical components of the induced magnetization produced by horizontal component of the geomagnetic field (labeled $\times \mathrm{Xix}$ and $\times \mathrm{Zix}$ in Figures 2021) can be obtained and can also be compared with the calculated field.

In the numerical calculations, the steel plate is meshed with 1445 nodes and 3957 tetrahedral elements. Using accelerated IEM, the number of surface integrals required is 9357, and the time for obtaining the system matrix is $15830.2 \mathrm{~s}$. In contrast, solving for the system matrix using conventional IEM requires 15828 surface integrals and $25198.3 \mathrm{~s}$. The three components of the magnetic flux are also calculated using Flux3D. In Figures 20 and 21, the normalized measured values (labeled Meax), the normalized values from conventional IEM (labeled NORMALX), the normalized values from accelerated IEM (labeled FAST $\times$ ) and the normalized values from simulations using Flux (labeled Flux $\times$ ) are displayed. Taking the values of the experiments as the baseline, the maximal errors using Flux3D, conventional IEM, accelerated IEM results are all less than $5.50 \%$.

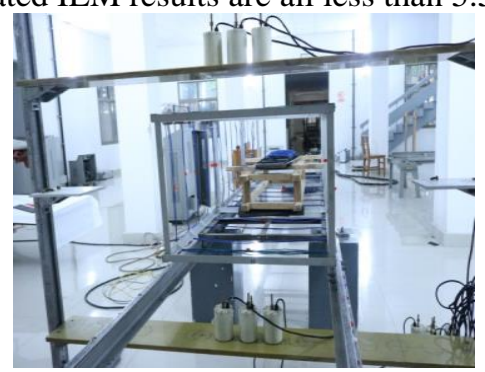

Figure 18 The measurement of the thin steel plate

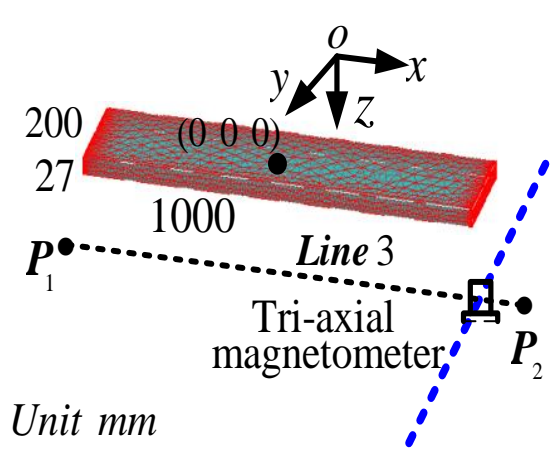

Figure 19 Map of the measurement of the steel plate

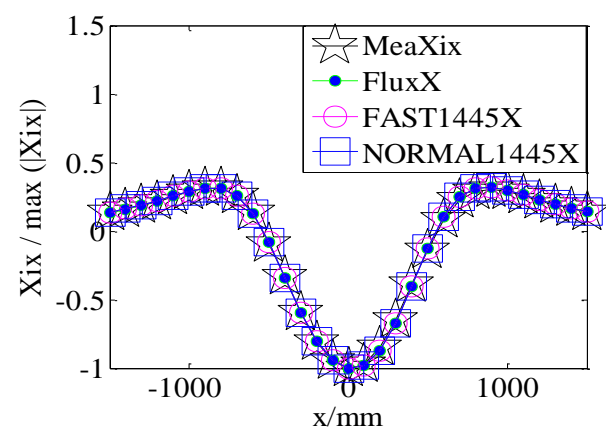

Figure 20 Normalized value of Xix of the thin steel plate

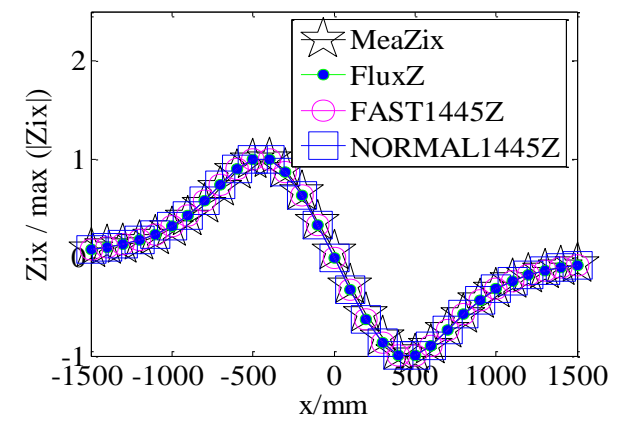

Figure 21 Normalized value of Zix of the thin steel plate

\section{Conclusion}

In this paper, based on the shared faces among the neighboring mesh elements, a new accelerated IEM algorithm is proposed that can reduce by a factor of $(N 3+N 4) /(2 N 3+N 4)$ (more than $40 \%$ ) the number of surface integrals that are required when the system matrix is constructed. The analytical, simulation and experimental examples here demonstrate the improved efficiency and computational accuracy of the proposed accelerated IEM. In future work, the efficiency of magnetostatic computation would be further improved by combining the proposed IEM with other fast computational methods such as ACA, FMM and parallel computing technique, which can be beneficial for large-scale magnetostatic field modeling.

\section{Acknowledgements}

Thank you for your cooperation in complying with these instructions. This work is supported in part by National 
Natural Science Foundation of China under grant No. 51377165 and No.41476153.

\section{References}

[1] J. J. Holmes., "Exploitation of a Ship's Magnetic Field Signatures (Synthesis Lectures on Computational Electromagnetics)", Morgan and Claypool Publishers, San Rafael, California, (2006).

[2] J. J. Holmes., "Reduction of a Ship's Magnetic Field Signatures (Synthesis Lectures on Computational Electromagnetics)", Morgan and Claypool Publishers, San Rafael, California, (2008).

[3] H. F. Pang, Q. Zhang, J. Li, S. T. Luo, D. X. Chen, M. C. Pang, F. L. Luo, "Improvement of vector compensation method for vehicle magnetic distortion field," Journal of magnetism and magnetic material, Vol. 353, (2014), pp. $1-5$.

[4] A. V. Kildishev and J. A. Nyenhuis, "External magnetic characterization of marine vehicles," OCEANS 2000 MTS, Vol.2, (2000), pp. 1145-1147.

[5] A. V. Kildishev, R. Kamondetdacha and J. A. Nyenhuis, "Prediction of the magnetic field beyond a rectangular array of sensors," Journal of Applied Physics, Vol.93, No.10, (2003), pp. 7074-7076.

[6] J. J. Gao, X. L. Zhu and W. B. Zhu, "Accurate calculating method of marine three-component geomagnetic field in shipboard measurement," Chinese Automation Congress 2015, Wuhan, China, November 27-29.

[7] M. Woloszyn, "Analysis of aircraft magnetic interference," International Joural of Applied Electromagnetics and Mechanics, Vol. 39, No.1-4, (2012), pp. 129-136.

[8] X. Brunotte, G. Meunier and J. Bongiraud, "Ship magnetization modeling by the finite element method," IEEE Trans. Mag., Vol.29, No.2, (1993), pp. 1970-1976.

[9] F. Damidau, B. Bandelier, and P. Penven, "A fast and precise determination of the static magnetic field in the presence of thin iron shells, " IEEE Trans. Mag., Vol.31, No.6, (1995), pp. 3491-3493.

[10] O. Chadebec, J. L. Coulomb, J. Bongiraud, G. Cauffet, P. L. Thiec, "Recent improvements for solving inverse magnetostatic problem applied to thin shells," IEEE Trans. Mag., Vol.38, No.2, (2002), pp. 1005-1008.

[11] O. Chadebec, J. L. Coulomb, V. Leconte, J. Bongiraud, G. Cauffet, "Modeling of static magnetic anomaly created by iron plates," IEEE Trans. Mag., Vol.36, No.4, (2000), pp.667-671.

[12] O. Chadebec, J. L. Coulomb, Cauffet, G., J. Bongiraud, "How to well pose a magnetization identification problem," IEEE Trans. Mag., Vol.39, No.43, (2003), pp.1634-1637.

[13] O. Chadebec, J. L. Coulomb, and F. Janet, "A review of magnetostatic moment method," IEEE Trans. Mag., Vol.42, No.4, (2006), pp.515-520.

[14] D. Rodger, P. J. Leonard and H. C. Lai, "Surface elements for modelling 3D fields around thin iron sheets," IEEE Trans. Mag., Vol.29, No.2, (1993), pp. 1483-1486.

[15] K. Z. Zhao, M. Vouvakis, J. F. Lee, "The adaptive cross approximation algorithm for accelerated method of moment computations of EMC problems," IEEE Trans. Mag., Vol.47, No.4, (2005), pp. 763-773

[16] G. H. Zhou, S. D. Liu, C. H. Xiao, and D. M. Liu, "Approach for a fast computation of induced magnetic field created by naval vessels, " International Journal of Applied Electromagnetics and Mechanics, Vol.47, No.3, (2015), pp. 753-765.

[17] M. Fang, K. H. Song, Z. X. Huang, X. L, Wu, "Fast electromagnetic simulation by parallel MoM implemented on CUDA," 2013 Proceedings of the International Symposium On Antennas \& Propagations, Nanjing, China, October 23-25.

[18] M. Zhang, L. Guo, "A fast algorithm for pre-procedure of method of moments," Journal of Nanjing University of Posts and Telecommunications:Natural Science Edition, Vol.30, No.5, (2010), pp. 95-97.

[19] M. W. Fan and W. L. Yan, "Integral equation method in electromagnetics", China Machine Press, Beijin, (1988).

[20] G. H. Zhou, C. H. Xiao, S. D. Liu, J. J. Gao, “3D magnetostatic field computation with hexahedral surface integral equation method," Tansactions of China Electrotechnical Society, Vol.24, No.3, (2009), pp. 1-7.

[21] Günter, L., "Electromagnetic Field Theory for Engineers and Physicists", Springer, New York, (2010). 